\title{
Parts function as perceptual organizational entities in infancy
}

\author{
Ashley Kangas • Nicole Zieber • Angela Hayden • \\ Ramesh S. Bhatt
}

Published online: 29 January 2013

(C) Psychonomic Society, Inc. 2013

\begin{abstract}
Both objects and parts function as organizational entities in adult perception. Prior research has indicated that objects affect organization early in life: Infants grouped elements located within object boundaries and segregated them from those located on different objects. Here, we examined whether parts also induce grouping in infancy. Five- and 6.5month-olds were habituated to two-part objects containing element pairs. In a subsequent test, infants treated groupings of elements that crossed part boundaries as novel, in comparison with groupings that had shared a common part during habituation. In contrast, the same arrangement of elements failed to elicit evidence of grouping in control conditions in which the elements were not surrounded by closed part boundaries. Thus, infants grouped and segregated elements on the basis of part structure. Part-based processing is a key aspect of many theories of perception. The present research adds to this literature by indicating that parts function as organizational entities early in life.
\end{abstract}

Keywords Object-based attention · Partperception · Infancy · Perceptual organization

A considerable amount of evidence indicates that objects constrain adults' visual functions. For example, objects function as bounded regions of attention, such that shifting of attention is more efficient within objects than between objects (e.g., Duncan, 1984; Egly, Driver, \& Rafal, 1994; Harrison \& Feldman, 2009; Hollingworth, Maxcey-Richard, \& Vecera, 2012; Reppa, Schmidt, \& Leek, 2012). Like objects, parts also guide adult perception. For instance, the spread of attention is constrained not only by object boundaries, but also by

A. Kangas $\cdot$ N. Zieber $\cdot$ A. Hayden $\cdot$ R. S. Bhatt $(\bowtie)$

Department of Psychology, University of Kentucky,

Lexington, KY 40506-0044, USA

e-mail: rbhatt@email.uky.edu part boundaries (e.g., Barenholtz \& Feldman, 2003; Singh \& Scholl, 2000 [reported in Singh \& Hoffman, 2001]; Vecera, Behrmann, \& Filapek, 2001; Watson \& Kramer, 1999). Presumably, the role of objects and parts as perceptual guides reflects organizational mechanisms that facilitate the generation of meaningful percepts from our environment.

Prior research indicates that objects function as organizational entities in infancy. The present research examined whether parts also guide organization early in life. The understanding of the development of part-based perceptual organization is important given that part-based processing is a key aspect of many perceptual theories (e.g., Biederman, 1987; Marr \& Nishihara, 1978). For instance, Biederman's model assumes that visual images are initially articulated into volumetric parts and object representations are created by the subsequent organization of these parts into perceptual wholes. Singh and Hoffman (2001) have suggested that a number of other perceptual and cognitive phenomena, including figure-ground segregation, shape similarity assessment, visual search, allocation of attention, and memory for shapes, are due to part representation.

Currently, not much is known about the role of parts as organizational entities during development. In fact, to date, only a few studies have addressed any aspect of part perception in early infancy. Bhatt, Hayden, Reed, Bertin, and Joseph (2006) found that, like adults (e.g., Xu \& Singh, 2002), 5- and 6.5-month-old infants find concave targets among convex distractors to be more salient than convex targets among concave distractors. This perceptual asymmetry indicates that concavities in the contours of images that signal part boundaries are salient to infants. In addition, infants are sensitive to the short-cut rule, which states that, all else being equal, object images are segmented into parts by using the shortest possible cuts across image boundaries (Bhatt, Hayden, Kangas, Zieber, \& Joseph, 2010). This sensitivity indicates that some of the rules that govern part segregation from images in adulthood 
are operational in infancy. Moreover, infants attend more to the shapes of parts than to the shapes of cavities (Hayden, Bhatt, Kangas, \& Zieber, 2011). Altogether, these studies indicate that at least some aspects of part perception seen in adults are functional during the first year of life.

There is also evidence suggesting that objects are organizational entities in infancy. Johnson and Gilmore (1998) found that cuing a part of an object affects 8-month-olds' subsequent saccades to a different part of the object versus another object and concluded that 8-month-olds' attention was object-based. Additionally, Bhatt, Hayden, and Quinn (2007) found that infants as young as 3.5 months of age group elements located in object-like regions in accord with the principle of common region (Palmer, 1992): Infants exposed to shapes A and B located in one rectangle and shapes $\mathrm{C}$ and $\mathrm{D}$ located in another rectangle subsequently found a mismatched grouping (e.g., $\mathrm{BC}$ ) to be novel, in comparison with an original grouping (AB). This result demonstrates that infants use object boundaries as regions to group visual elements (also see Hayden, Bhatt, \& Quinn, 2008; Kangas, Zieber, Hayden, Quinn, \& Bhatt, 2012).

Prior research thus indicates that objects can function as organizational units in infancy and that at least some aspects of part perception seen in adults are already evident during the first year of life. The question then arises as to whether parts also function as organizational entities in infancy, as they do in adulthood. To address this question, we examined whether object parts act as boundaries that induce grouping and segregation in infancy. If so, it would indicate that a key characteristic of adult perception - namely, the role of parts as perceptual organizers - is developed quite early in life.

Currently, no theory of perceptual development directly addresses part-based organization early in life. However, Kellman's (1996, 2003; Kellman \& Arterberry, 1998) theory concerning the development of edge-based processing is relevant to the present research because the part structure of the stimuli used in this study was signaled by edge discontinuities. Kellman's model proposes a distinction between edgeinsensitive (EI) and edge-sensitive (ES) processes. The EI process is thought to be available at birth and responds to motion information, while the "rich" ES system is thought to become functional only after 6-7 months of age and to respond to edge information such as good continuation. In the present study, part structure was signaled by concavities in contours of static images, which would have to be processed by the ES system. Thus, the Kellman model predicts that infants younger than 6 months of age will not exhibit evidence of part-based organization, whereas older infants with more developed ES systems will be able to process edge information and, therefore, potentially be able to derive part structure and organize accordingly. We tested these predictions in the present study by examining the performance of 5- and 6.5month-old infants.

\section{Method}

\section{Participants}

Thirty-two 5-month-olds (mean age $=151.19$ days, $S D=6.06$; 15 female) and twenty-four 6.5-month-olds (mean age = 205.54 days, $S D=8.39 ; 8$ female) were recruited through birth announcements and from a local hospital. The infants were predominately Caucasians from middle-class families. Data from 10 additional infants were excluded due to parental interference $(n=2)$, fussiness $(n=4)$, failure to sample both test stimuli $(n=1)$, or position preference $(90 \%$ or greater looking to one side; $n=3$ ).

\section{Stimuli}

Infants in the experimental condition were exposed to images of objects with two parts, each containing a pair of elements (Fig. 1). As in prior studies (e.g., Barenholtz \& Feldman, 2003; Singh \& Hoffman, 2001), parts were delineated by concavities; that is, a concave contour inflexion was assumed to indicate a boundary between parts, while a convex inflexion was not. One pair of elements always shared a common part, while the other pair was located on the other part. Note that the elements within parts were also divided by an inflexion point, but it was convex and, therefore, did not signal a part boundary. If infants fail to group elements within parts and segregate them from those on other parts, it would suggest that any inflexion point, concave or convex, affects grouping or, alternatively, that infants are insensitive to inflexion points in general. On the other hand, if infants group elements on the basis of parts despite the presence of the convexity within parts, it would indicate that contour inflexions that signal part boundaries have a special status and affect perceptual organization (Barenholtz \& Feldman, 2003).

Across trials, the location of the elements varied from one part to the next in a counterbalanced manner in order to prevent the association of particular elements with particular locations in the image (Fig. 1). The element pairings were thus defined by the sharing of a common part region, although this part varied from one trial to the next. Each element subtended approximately $1.27^{\circ}$, and the space between them subtended $2.56^{\circ}$. The distances between the elements were such that the element that was closest to the part boundary was as far from the other element within the same part as the shape that occupied the nearest position on the other part. In other words, the distances between the within-part elements and the closest across-part elements were equated, such that element pairings could not be formed due to proximity. Overall, the whole image subtended $10.79^{\circ} \times 16.69^{\circ}$, while each individual part was $10.79^{\circ} \times 8.33^{\circ}$ in size. 
Trial 1
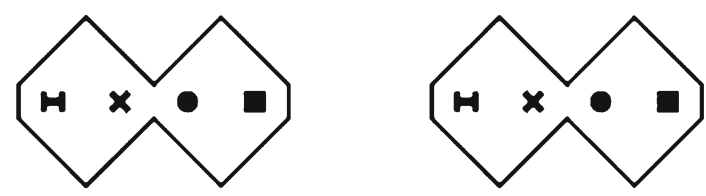

Trial 2
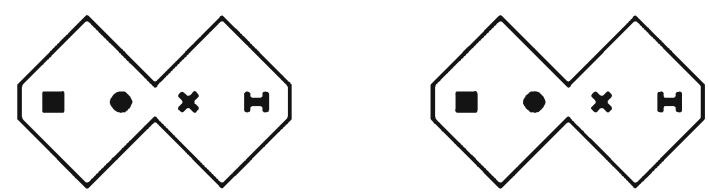

Test
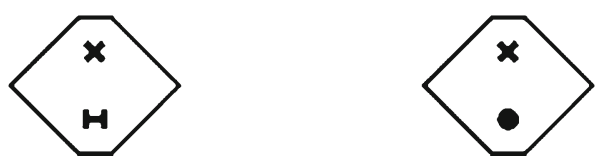

Fig. 1 Examples of the stimuli used in the experimental condition of the present study. Infants were habituated to two pairs of shape elements contained within parts, with one pair sharing a part and the other a different part. Infants were then tested for their preference between pairs of shapes that had shared parts during habitation versus pairs of shapes that had been on different parts during habituation. There was an orientation change from habituation to test. The pairs of elements that made up the within-part and between-part patterns during habituation were counterbalanced across infants; thus, within each age group, each pair of elements equally often reflected familiar and novel pairings during the test

Two different combinations of two pairs of elements were derived from the shapes $\mathrm{H}, \mathrm{X}, \mathrm{O}$, and square. Half of the infants were habituated with one combination, while the other half were habituated to the other set. After habituation, infants were tested for a preference between a within-part pairing and a between-part pairing (Fig. 1). The test stimuli were counterbalanced across infants such that each pair of elements appeared equally often as a within-part and a between-part pairing during the test. Thus, any preference during the test could be due only to the status of the pairs during habituation (i.e., whether a pair shared a common part or not), rather than to any a priori stimulus preferences. During the test, the stimuli were oriented differently than during habituation - that is, vertically (Fig. 1). This change was intended as a test of infants' abilities to generalize grouping to a novel situation and matched the tests in prior studies that were conducted to examine grouping based on object boundaries (Bhatt et al., 2007). The left/right location of the familiar/novel grouping on the computer monitor during the test was counterbalanced across infants, and this location was switched from one trial to the next.

Infants in the control condition saw the same stimulus arrangements as those in the experimental condition, except that the patterns they saw were not composed of enclosed parts (Fig. 2a, b). Two different versions of the control stimuli were used. In one, the individual contours that made up the parts in the experimental condition remained intact but were pulled apart such that they no longer represented enclosed regions (Fig. 2a). In the other, the contours were shortened so that they no longer intersected each other to form enclosed regions (Fig. 2b). We used these two types of control stimuli because each kind of change disrupted the enclosed nature of the stimuli used in the experimental condition in a different way. In the case shown in Fig. 2a, the overall size of the stimuli differed from those used in the experimental condition, while the lines that formed the contour remained the same; in the other case (Fig. 2b), the overall extent remained the same, but the amount of contour was less than in the experimental condition. However, preliminary analyses revealed no difference in the performance in the two control conditions; consequently, infants in the control conditions were treated as a unified group in the analyses reported in the Results section.

\section{Apparatus}

Infants were seated approximately $45 \mathrm{~cm}$ in front of a $50-\mathrm{cm}$ computer monitor. Floor-to-ceiling black cloth ensured that the infant saw only the monitor during the test session. The monitor was the only source of light in the test chamber. A camera located on top of the monitor and a DVD recorder were used to document infants' look direction and duration.

\section{Procedure}

We used a standard infant-control procedure (e.g., Bhatt et al., 2007; Hayden et al., 2008). Each trial began with the presentation of an attention getter, a rapidly changing set of colored shapes, in the middle of the computer monitor. Once the infant fixated it, two identical images depicting pairs of shape elements sharing a common part were presented in the experimental condition and its corresponding control version in the control condition (see Figs. 1 and 2). The images remained on the screen until the infant looked away for $2 \mathrm{~s}$ or until a total of $60 \mathrm{~s}$ had accumulated. Such habitation trials continued until the mean look duration during three consecutive trials was less than $50 \%$ of the mean look duration during the first three trials. Two test trials followed habituation; in each, the two test stimuli (Figs. 1 and 2) were presented side-by-side for $10 \mathrm{~s}$.

Coding was conducted offline by an observer who was unaware of the left/right location of the novel stimuli. 
Fig. 2 Examples of the stimuli used in the control condition of the present study. The element arrangements were the same as in the experimental condition. However, the lines that formed the external contour were displaced (a) or shortened (b) in such a way that the stimuli were no longer enclosed images depicting two-part objects

\section{a}

Trial 1

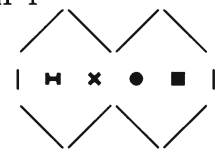

Trial 2

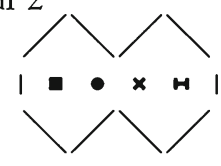

Test

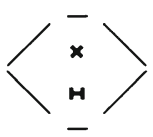

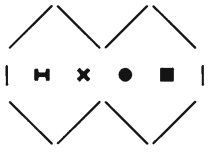

Trial 1
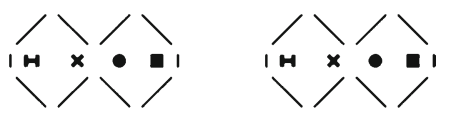

Trial 2
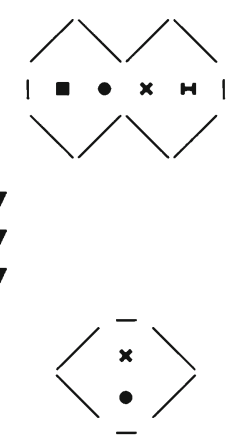
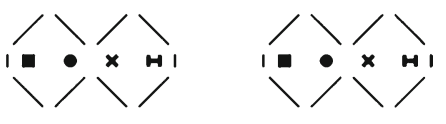

Test

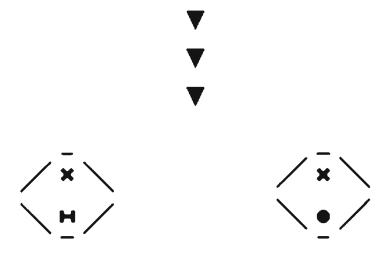

Another naïve observer coded the performance of 14 infants in order to assess coding reliability. The Pearson correlation between the two observers' scores was .97.

\section{Results}

Table 1 displays the infants' look durations during habituation. A condition (experimental, control) $\times$ age $(5$, 6.5 months) $\times$ trial blocks (first three, last three) ANOVA revealed only a significant effect of trial blocks, $F(1,52)=$ $161.95, p<.001$. All other main and interaction effects were nonsignificant (all $p \mathrm{~s}>.45$ ). Similarly, an ANOVA failed to reveal any differences between age groups or conditions or an interaction in the number of trials needed for habituation (all $p \mathrm{~s}>.65$ ). Thus, infants at both ages and in both conditions habituated before being tested (as required by the procedure), and there was no evidence indicating differences in habituation patterns across age groups or conditions.

As in prior studies (Bhatt et al., 2007; Hayden et al., 2008; Kangas et al., 2012), we used a novelty preference measure to assess discrimination during the test. This measure was derived by dividing the total look duration to the novel patterns during the two tests by the total look durations to both the familiar and novel patterns and by multiplying this ratio by 100 . A group mean novelty preference score that is significantly greater than $50 \%$ indicates discrimination, whereas a score that is not different from $50 \%$ indicates a lack of discrimination.

The mean novelty preference scores are displayed in Table 1 . An age $(5,6.5$ months $) \times$ condition (experimental, control) ANOVA revealed a main effect of condition, $F(1,52)=5.25$, $p<.03, n_{\mathrm{p}}{ }^{2}=.09$. Neither the age main effect, $F(1,52)<1$, $p=.88$, nor the interaction, $F(1,52)=1.07, p=.31$, was significant. Thus, the score in the experimental condition was significantly greater than the score in the control condition, and there was no evidence indicating that this differed as a function of age. Individual sample $t$-tests comparing the scores against the chance level of $50 \%$ indicated that the scores of both the 5 month-olds and the 6.5-month-olds in the experimental condition were significantly greater than $50 \%$, but the scores in the control conditions were not (Table 1). These results indicate that infants detected a change when elements sharing common parts were regrouped into novel combinations (experimental condition) but failed to detect the same change when the contours surrounding the pairs did not signals parts (control condition). Thus, infants exhibited grouping based on part structure.

\section{Discussion}

Five- and 6.5-month-old infants grouped shape elements that were located on parts of an object and segregated them from elements located on other parts. This result indicates that parts function as organizational entities that guide perceptual grouping in infants. Thus, it appears that a fundamental organizational mechanism that facilitates adult perception is operational quite early in life.

Part-based processing is a key aspect of many perceptual theories (e.g., Biederman, 1987; Marr \& Nishihara, 1978) and is thought to underlie a number of perceptual and cognitive phenomena, including figure-ground segregation, shape similarity assessment, visual search, allocation of attention, and memory for shapes (Singh \& Hoffman, 2001). The present work adds to this literature by indicating that parts function as entities that induce grouping early in life.

The present findings are inconsistent with accounts of perceptual development that posit that edge-based processing is a late-developing function. As was discussed in the introduction, Kellman's (1996; Kellman \& Arterberry, 1998) model assumes 
Table 1 Mean (and standard error) of number of trials to habituate, fixation duration during habituation trials, and percent preference for the novel pairing of elements exhibited during test trials

\begin{tabular}{|c|c|c|c|c|c|c|}
\hline & \multirow[t]{3}{*}{$N$} & \multicolumn{3}{|l|}{ Habituation } & \multirow{2}{*}{\multicolumn{2}{|c|}{$\frac{\text { Test }}{\text { Preference for novel pairing (\%) }}$}} \\
\hline & & \multirow[t]{2}{*}{ Number of trials } & \multirow[t]{2}{*}{ First three trials (s) } & \multirow[t]{2}{*}{ Last three trials (s) } & & \\
\hline & & & & & $\mathrm{M}(\mathrm{SE})$ & $t(\mathrm{vs} .50 \%$ chance $)$ \\
\hline \multicolumn{7}{|l|}{ 5-month-olds } \\
\hline Experimental & 16 & $9.50(.89)$ & 32. 41(3.99) & $11.34(1.73)$ & $54.69(2.05)$ & $2.30^{* *}$ \\
\hline Control & 16 & $8.82(.92)$ & $30.35(3.48)$ & $13.42(1.56)$ & $51.74(1.86)$ & 0.93 \\
\hline \multicolumn{7}{|c|}{ 6.5-month-olds } \\
\hline Experimental & 12 & $9.17(1.39)$ & $30.34(4.79)$ & $11.71(1.72)$ & $56.76(3.19)$ & $2.12^{*}$ \\
\hline Control & 12 & $9.42(1.34)$ & $31.25(3.88)$ & $12.10(1.63)$ & $48.96(2.32)$ & -0.44 \\
\hline
\end{tabular}

${ }^{*} p<.06$, two-tailed; $* * p<.05$, two-tailed

that the ES system is not functional before 6-7 months of age. However, 5-month-olds in the present study exhibited partbased organization, with parts signaled by discontinuities in static image contours. Thus, the present findings argue against the proposal of a late-developing ES system. In this respect, the results are consistent with other reports of early development of edge-based processing (e.g., Bhatt et al., 2007; Ghim, 1990; Hayden et al., 2008; Quinn \& Bhatt, 2005).

Note that the stimuli used in the present study were such that the distances between the within-part and between-part test elements were the same, such that proximity could not have been used to group elements. Along with other studies that have demonstrated grouping based on object boundaries rather than proximity (e.g., Bhatt et al., 2007), the present study thus indicates that absolute spatial positions in an image do not necessarily drive organization. Rather, even early in life, the visual system takes into consideration contextual information, including part structure, to organize images.

Another point to note is that even the elements located within parts in the present study were divided by an inflexion point in the surrounding contour (Fig. 1). In terms of the degree of inflexion, this convexity was equivalent to the concavity that signaled the part boundary. Yet infants grouped elements within parts and segregated elements from different parts. Thus, the nature of the inflexion point is important in infancy: Concavities that signal parts have a special status and induce grouping of elements located on the same side of the inflexion and segregation from those on the different side of the inflexion, whereas convexities that do not signal parts do not induce similar organization. This finding is consistent with those of Barenholtz and Feldman (2003) with adults, who found that concavities are more significant organizational boundaries than are convexities.

The lack of grouping by infants in the control conditions of the present study is also noteworthy. The stimuli used in the control conditions were such that part regions could have been constructed by the infants and used to organize information through illusory contour formation. The failure of grouping in the control condition suggests that infants could not bridge the contour gaps to construct illusory regions. In prior studies, infants as young as 3 months of age exhibited evidence of perceiving illusory contours (e.g., Bremner, Slater, Johnson, Mason, \& Spring, 2012; Ghim, 1990; Hayden et al., 2008). However, other studies indicate that illusory contour formation is not evident until later in life (e.g., Csibra, 2001), and its development continues well into childhood (Palomares, Landau, \& Egeth, 2008). The contradictory evidence in infant studies indicates that illusory contours are perceived by young infants only if there is sufficient information to support contour formation. For instance, in Bremner et al. (2012), while 4-month-olds perceived illusory occluding surfaces when both occluding edges and the occlusion of background texture elements were present at the same time, they failed to do so if only one of these cues was present. It appears that the level of information in the control conditions of the present study was similarly not enough for infants to perceive illusory part regions.

Future studies will have to address the exact mechanisms that underlie the functioning of parts as organizational units in infancy. Many studies of object- and part-based organization in adulthood have concentrated on the spatial spread of attention within versus outside objects/parts (see Reppa et al., 2012, for a review). As was noted earlier, the general finding is that object and part borders function as attentional boundaries. We did not assess attentional effects in the present study. It is thus not clear whether the grouping of elements based on part structure in the present study was driven by attentional mechanisms or by some other process.

In summary, part structure guided 5- and 6.5-month-olds' grouping of elements in the present study. In combination with prior studies indicating that object boundaries guide perceptual grouping in infancy (e.g., Bhatt et al., 2007; 
Hayden et al., 2008; Kangas et al., 2012), this result demonstrates that both parts and objects function as organizational entities in infancy, as they do in adulthood. Thus, even early in life, the human visual system is able to utilize mechanisms that constrain perception and guide the derivation of a functional representation of the environment.

Author Note This research was supported by a grant from NSF (BCS-1121096). We thank the infants and the parents who participated in this study. Address correspondence to Ramesh S. Bhatt, Department of Psychology, University of Kentucky, Lexington, KY 40506-0044, U.S.A. Email: rbhatt@email.uky.edu.

\section{References}

Barenholtz, E., \& Feldman, J. (2003). Visual comparisons within and between object parts: Evidence for a single-part superiority effect. Vision Research, 43, 1655-1666.

Bhatt, R. S., Hayden, A., Kangas, A., Zieber, N., \& Joseph, J. E. (2010). Part perception in infancy: Sensitivity to the short-cut rule. Attention, Perception, \& Psychophysics, 72(4), 1070-1078.

Bhatt, R. S., Hayden, A., \& Quinn, P. C. (2007). Perceptual organization based on common region in infancy. Infancy, 12, 147-168.

Bhatt, R. S., Hayden, A., Reed, A., Bertin, E., \& Joseph, J. E. (2006). Infants' perception of information along object boundaries: Concavities versus convexities. Journal of Experimental Child Psychology, 94, 91-113.

Biederman, I. (1987). Recognition-by-components: A theory of human image understanding. Psychological Review, 94, 115-147.

Bremner, J. G., Slater, A. M., Johnson, S. P., Mason, U., \& Spring, J. (2012). Illusory contour figures are perceived as occluding contours by 4-month-old infants. Developmental Psychology, 48, 398-405.

Csibra, G. (2001). Illusory contour figures are perceived as occluding surfaces by 8 -month-old infants. Developmental Science, 4, F7F11.

Duncan, J. (1984). Selective attention and the organization of visual information. Journal of Experimental Psychology. General, 113 (4), 501-517.

Egly, R., Driver, J., \& Rafal, R. D. (1994). Shifting visual attention between objects and locations: Evidence from normal and parietal lesion subjects. Journal of Experimental Psychology. General, 123, 161-177.

Ghim, H. R. (1990). Evidence for perceptual organization in infants: Perception of subjective contours by young infants. Infant Behavior \& Development, 13, 221-248.

Harrison, S. J., \& Feldman, J. (2009). Perceptual comparison of features within and between objects: A new look. Vision Research, 49, 2790-2799.

Hayden, A., Bhatt, R. S., Kangas, A., \& Zieber, N. (2011). Parts, cavities, and object representation in infancy. Journal of Experimental Psychology. Human Perception and Performance, 37, 314-317.
Hayden, A., Bhatt, R. S., \& Quinn, P. C. (2008). Perceptual organization based on illusory regions in infancy. Psychonomic Bulletin and Review, 15, 443-447.

Hollingworth, A., Maxcey-Richard, A. M., \& Vecera, S. P. (2012). The spatial distribution of attention within and across objects. Journal of Experimental Psychology. Human Perception and Performance, 38, 135-151.

Johnson, M. H., \& Gilmore, R. O. (1998). Object-centered attention in 8-month-old infants. Developmental Science, 1, 221-225.

Kangas, A., Zieber, N., Hayden, A., Quinn, P. C., \& Bhatt, R. S. (2012). Transfer of associative grouping to novel perceptual contexts in infancy. Attention, Perception, \& Psychophysics, 73(8), 2657-2667.

Kellman, P. J. (1996). The origins of object perception. In R. Gelman \& T. K. Au (Eds.), Perceptual and cognitive development (pp. 348). San Diego: Academic Press.

Kellman, P. J. (2003). Visual perception of objects and boundaries: A four-dimensional approach. In R. Kimchi, M. Behrmann, \& C. R. Olson (Eds.), Perceptual organization in vision: Behavioral and neural perspectives (pp. 155-201). Mahwah, NJ: Lawrence Erlbaum Associates.

Kellman, P. J., \& Arterberry, M. E. (1998). The cradle of knowledge: Development of perception in infancy. Cambridge, MA: MIT press.

Marr, D., \& Nishihara, H. K. (1978). Representation and recognition of three dimensional shapes. Proceedings of the Royal Society of London, Series B, 200, 269-294.

Palmer, S. E. (1992). Common region: A new principle of perceptual grouping. Cognitive Psychology, 24, 436-447.

Palomares, M., Landau, B., \& Egeth, H. (2008). Visuospatial interpolation in typically developing children and in people with Williams Syndrome. Vision Research, 48, 2439-2450.

Quinn, P. C., \& Bhatt, R. S. (2005). Good continuation affects discrimination of visual pattern information in young infants. Perception \& Psychophysics, 67, 1171-1176.

Reppa, I., Schmidt, W. C., \& Leek, E. C. (2012). Successes and failures in producing attentional object-based cuing effects. Attention, Perception, \& Psychophysics, 74, 43-69.

Singh, M., \& Hoffman, D. D. (2001). Part-based representations of visual shape and implications for visual cognition. In T. F. Shipley \& P. J. Kellman (Eds.), Advances in psychology: Vol.130. From fragments to objects-segmentation and grouping in vision (pp. 37-69). New York: Elsevier. (pp. 401-459). Mahwah, NJ: Erlbaum.

Singh, M., \& Scholl, B. (November, 2000). Using attentional cueing to explore part structure. Poster presented at the Annual Symposium of Object Perception and Memory, New Orleans, Louisiana.

Vecera, S. P., Behrmann, M., \& Filapek, J. C. (2001). Attending to the parts of a single object: Part-based selection limitations. Perception \& Psychophysics, 63, 308-321.

Watson, S. E., \& Kramer, A. F. (1999). Object-based visual selective attention and perceptual organization. Perception \& Psychophysics, 61, 31-49.

$\mathrm{Xu}, \mathrm{Y} .$, \& Singh, M. (2002). Early computation of part structure: Evidence from visual search. Perception \& Psychophysics, 64 (7), 1039-1054. 